\title{
Enseigner la pensée informatique avec Kniwwelino : Étude exploratoire de terrain en milieu scolaire
}

\section{Teaching Computational Thinking with Kniwwelino: Exploratory Field Study in School Settings}

\author{
Lou Schwartz \\ Valérie Maquil \\ Laurence Johannsen \\ Christian Moll \\ firstname.lastname@list.lu \\ Luxembourg Institute of Science and Technology (LIST) \\ Esch-sur- Alzette, Luxembourg
}

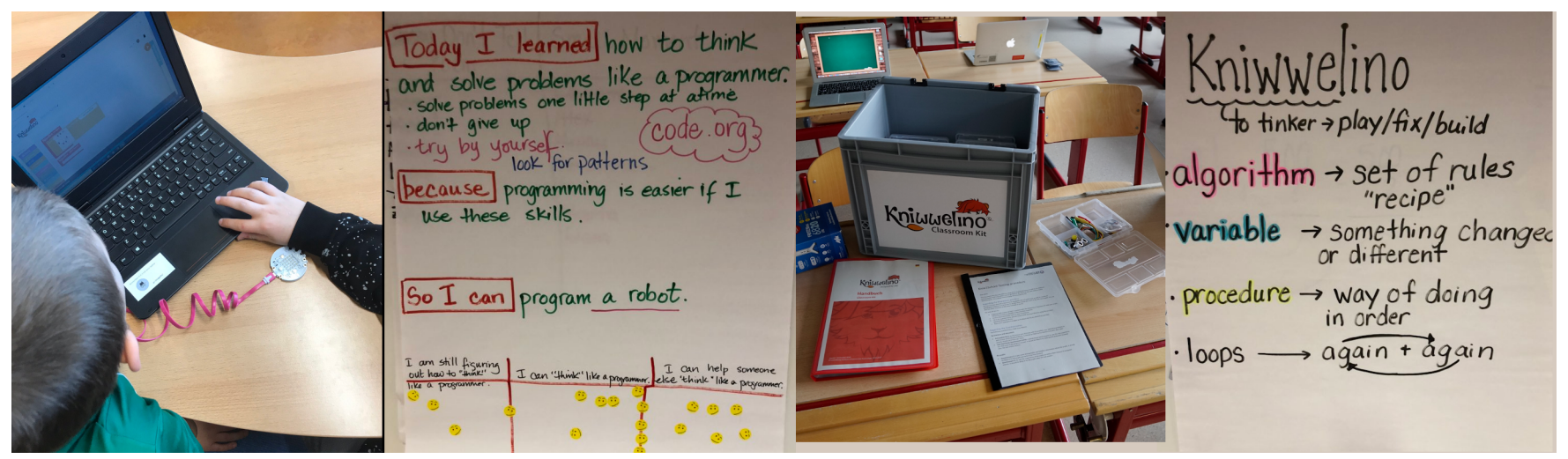

Figure 1: Utilisation du Kniwwelino Classroom Kit dans les écoles pilotes pour enseigner la pensée informatique.

\begin{abstract}
We are presenting the results of a study carried out with six primary and secondary schools in Luxembourg, pilots in introducing the Kniwwwelino Classroom kit (KCK) in their teaching of programming and computational thinking to children. The aim of the study was to explore: (1) how teachers appropriate this kind of tool for teaching computational thinking and programming, (2) whether the KCK is suitable for this purpose, and (3) the user experience of the KCK. To these ends, we used several data collection tools through a mainly qualitative exploratory approach: semi-guided interviews, diaries and several questionnaires. We have learned that: (1) the KCK is versatile enough to adapt to different environments, objectives and pedagogical approaches and thus offers a great deal of freedom to teachers, (2) the playful and tangible approach of the KCK seems more adapted to introducing programming and enables pupils to develop problem-solving, collaboration and creativity skills, and

Permission to make digital or hard copies of all or part of this work for personal or classroom use is granted without fee provided that copies are not made or distributed for profit or commercial advantage and that copies bear this notice and the full citation on the first page. Copyrights for components of this work owned by others than ACM must be honored. Abstracting with credit is permitted. To copy otherwise, or republish, to post on servers or to redistribute to lists, requires prior specific permission and/or a fee. Request permissions from permissions@acm.org.

IHM '20.21, April 13-16, 2021, Virtual Event, France

(c) 2021 Association for Computing Machinery.

ACM ISBN 978-1-4503-8362-2/21/04 .\$15.00

https://doi.org/10.1145/3450522.3451250
\end{abstract}

(3) if the user experience has been positive for both teachers and students, this first KCK prototype is still addressing intermediate computer-skilled teachers.

\section{CCS CONCEPTS}

- Applied computing $\rightarrow$ Interactive learning environments; - Human-centered computing $\rightarrow$ Field studies.

\section{KEYWORDS}

Interactive Learning Environments, Field Studies, Computational Thinking, Programming, K-12 Education

\section{RÉSUMÉ}

Nous présentons ici les résultats d'une étude pilote menée avec six écoles primaires et secondaires au Luxembourg, pionnières pour utiliser le Kniwwelino Classroom kit (KCK) dans leur enseignement de la programmation et la pensée informatique aux enfants. L'étude visait à explorer : (1) comment les enseignants s'approprient ce type d'outil pour enseigner la pensée informatique et de la programmation, (2) si le KCK est adapté à cet usage, (3) et l'expérience utilisateur du KCK. Pour ce faire, nous avons utilisé plusieurs outils de collecte d'information à travers une approche exploratoire principalement qualitative : entretiens semi-guidés, journaux de bord et plusieurs questionnaires. Nous avons appris que (1) le KCK est assez versatile pour s'adapter à différents milieux, objectifs et approches 
pédagogiques, offrant ainsi une grande liberté aux enseignants, (2) que l'approche ludique et tangible du KCK semble plus adaptée à la découverte ou à l'initiation à la programmation et permet aux élèves de développer leur capacité à résoudre des problèmes, collaborer et être créatifs et (3) que si l'expérience utilisateur a été positive pour les enseignants et les élèves, ce premier prototype du KCK s'adresse encore à des enseignants à l'aise avec l'informatique.

\section{MOTS-CLÉS}

Environnements d'Apprentissage Interactifs, Étude de Terrain, Pensée Informatique, Programmation, Éducation

\section{ACM Reference Format:}

Lou Schwartz, Valérie Maquil, Laurence Johannsen, and Christian Moll. 2021. Enseigner la pensée informatique avec Kniwwelino : Étude exploratoire de terrain en milieu scolaire: Teaching Computational Thinking with Kniwwelino: Exploratory Field Study in School Settings . In 32e Conférence Francophone sur l'Interaction Homme-Machine (IHM '20.21), April 13-16, 2021, Virtual Event, France. ACM, New York, NY, USA, 11 pages. https://doi.org/10.1145/3450522.3451250

\section{INTRODUCTION}

De nos jours, il est indispensable de comprendre l'informatique pour vivre dans notre société de l'information. Les compétences liées à l'informatique ont tendance à devenir un prérequis pour tout emploi. L'enseignement de la pensée informatique ou computationnelle devient indispensable. La pensée informatique, est définie par Wing comme "le processus de pensée impliqué dans la formulation d'un problème et l'expression de sa (ses) solution(s) de manière à ce qu'un ordinateur - humain ou machine - puisse le réaliser efficacement." [23]. Angeli définit six compétences qui composent la pensée informatique (PI) : l'abstraction (décider quelle information à propos d'une entité/d'un objet conserver et quelle information ignorer), la généralisation (formuler une solution en termes génériques afin qu'elle puisse être appliquée à des problèmes différents), la décomposition (découper un problème complexe en plus petits éléments plus simples à comprendre et à résoudre, l'algorithmique (concevoir un ensemble d'opérations / actions, étape par étape, afin de résoudre un problème), le débogage (identifier, supprimer et corriger les erreurs) [1].

$\mathrm{Au}$ Luxembourg, l'enseignement de la PI a été introduit dès septembre 2020 dans les classes de cycle 4 de l'école fondamentale (primaire), afin d'être progressivement étendu dans les autres cycles de l'école fondamentale ainsi qu'au lycée. Les compétences en PI sont enseignées conjointement avec les compétences mathématiques ou de manière transversale dans différentes disciplines comme les langues, l'éveil aux sciences ou l'éveil à la création. Au lycée, un nouveau cours en sciences numériques débutera en septembre 2021.

Dernièrement plusieurs plateformes de développement à prix abordable ont vu le jour : Arduino [2], BBC Micro:bit [3], Calliope mini [5], Lego Mindstorms [15], littleBits [4], MakeMe [11], Talkoo [12] et Kniwwelino [17]. L'objectif de ces plateformes est de proposer un moyen simple et engageant de fabriquer et programmer ses objets électroniques en s'amusant, afin de développer la PI des enfants. Mais comment utiliser ces plateformes dans un contexte scolaire afin d'enseigner la programmation et la PI ? Est-ce que ces plateformes sont adaptées à cet objectif ? C'est ce que nous allons discuter dans ce papier à travers une étude menée auprès de plusieurs écoles pilotes au Luxembourg lors de leur utilisation de Kniwwelino pour enseigner l'informatique en primaire et secondaire. Cette étude vise notamment à observer l'utilisation du KCK en classe par des enseignants et les pratiques qui en émergent.

\section{ENSEIGNER LA PENSÉE INFORMATIQUE EN CLASSE}

\subsection{Outils existants supportant l'enseignement de la pensée informatique}

Il existe différents types d'outils pour supporter l'apprentissage de la PI. Ching et al. ont listé les suivants [6] : les jouets programmables (comme Think \& Learn Code-a-pillar ${ }^{\mathrm{TM}}$ ), les kits robotiques (comme LEGO ${ }^{\circledR}$ Mindstorms EV3), les jeux de société (comme Robot Turtles Game), les outils tangibles augmentés (comme Osmo Coding Awbie), les outils de programmation d'applications/sites web (comme code.org), les outils d'animation/de développement de jeux (comme Scratch). Auxquels on peut ajouter les microcontrôleurs sur lesquels connecter des extensions électroniques et programmation à l'écran, comme Micro:bit ou Kniwwelino. Ils sont aussi puissants et polyvalents que les outils de développement d'animation, mais ils permettent aussi de s'initier à l'électronique.

Les langages de programmation visuelle sont les outils les plus utilisés pour l'enseignement de la PI [10]. Lye et Koh recommandent l'utilisation de langage de programmation visuelle pour apprendre la PI à des enfants de primaire et secondaire (K-12), afin de leur permettre de se concentrer sur la logique plutôt que sur la syntaxe [16]. L'utilisation de microcontrôleurs associé à de la programmation visuelle L'aspect visuel et parfois tangible des outils d'apprentissage permet d'intégrer plus facilement la PI grâce à la visualisation concrète des effets du programme "écrit" par l'élève, surtout pour les dimensions test et débogage[16, 19]. De plus, la possibilité d'un retour immédiat et tangible dans le monde réel semble bénéfique pour l'apprentissage de la PI en jouant fortement sur la motivation et l'engagement [19]. La réalisation de jeux et de projets donne une vue sur la conception et la réalisation d'applications, ce qui permet également d'améliorer l'engagement des élèves [19].

\section{2 Études menées sur l'enseignement de la pensée informatique en classe}

La plateforme de programmation visuelle la plus utilisée pour enseigner la PI est Scratch [10]. Son utilisation pour l'introduction à la programmation chez les enfants a été évaluée dans plusieurs études. Saez et. al [18] valident la pertinence de l'apprentissage de la programmation avec une plateforme de programmation visuelle (Scratch) auprès d'élèves d'école primaire (5th et 6th grade) à travers une étude menée auprès de 107 élèves dans cinq écoles différentes en Espagne sur une durée de deux ans lors des cours de science et d'art. L'utilisation de Scratch a eu comme conséquences d'amplifier la motivation, le plaisir, l'engagement et l'enthousiasme des élèves montrant des améliorations liées à la PI et aux pratiques informatiques.

Dans [13], l'apprentissage de la programmation avec Scratch a été évalué en demandant à 11 élèves de 5 th grade, 9 élèves de 8th grade et 16 élèves de 2 nd de suivre un cursus d'initiation 
à la programmation avec Scratch pendant cinq mois. La formation portait sur une introduction à Scratch, une prise en main par l'expérimentation, des leçons guidées avec introduction de nouveau contenu, travail en groupe sur des exemples et résolution de tâches individuelles en mettant l'accent sur la résolution de problèmes. Dans cette étude Scratch était souvent la première expérience de la programmation pour les participants. A la suite de cette utilisation de Scratch en classe, les élèves devaient répondre à un questionnaire avec une échelle de Likert à 5 niveaux (de 1 je ne suis pas du tout d'accord à 5 je suis totalement d'accord). Les réponses au questionnaire sont les suivantes : Scratch est intéressant $(3,81)$, Scratch est facile à utiliser $(3,94)$, Scratch était intéressant dans l'utilisation faite $(4,22)$, Scratch m'a motivé à travailler $(3,25)$, Scratch a été une expérience positive pour moi $(4,06)$, c'était amusant de travailler avec Scratch $(4,03)$, Programmer est difficile $(3,31)$, je savais toujours quoi faire avec Scratch $(3,08)$.

Une étude menée par Shina [20] montre que $60 \%$ des élèves interrogés ont trouvé facile d'utiliser Scratch. L'étude comprenait 27 enfants de 9 à 12 ans lors d'un atelier d'introduction à Scratch d'une heure et demie dans un établissement d'enseignement nonformel.

Sentance et al. ont étudié l'utilisation de la carte BBC Micro:bit pour enseigner la PI dans des écoles en Angleterre [22]. Cette étude met en avant que l'aspect tangible accentue la motivation et pousse à la collaboration et à la créativité, de plus elle permet aux élèves de mieux comprendre ce qu'il se passe dans le programme.

\section{LE KNIWWELINO CLASSROOM KIT (KCK)}

Kniwwelino ${ }^{\circledR}$ est un environnement créatif d'apprentissage de la programmation et de l'électronique [17]. Spécialement conçu pour les jeunes enfants (à partir de 8 ans), il permet de découvrir les bases de la programmation. L'environnement Kniwwelino comprend une carte électronique basée sur les technologies Arduino ${ }^{\circledR}$ et $\mathrm{Wi}-\mathrm{Fi}^{\mathrm{TM}}$ et une interface ludique et visuelle en ligne pour programmer en agrégeant des blocs à la manière d'un puzzle. Des extensions, connectées à la carte, permettent de découvrir l'électronique. L'intérêt de Kniwwelino, par rapport à BBC Micro:bit par exemple, est notamment sa connexion Wi-Fi et l'utilisation de MQTT pour échanger des messages entre les différentes cartes ou avec d'autres objets connectés ou services web. Devant le succès rencontré par Kniwwelino auprès des enfants et de leurs encadrants lors de foires, ateliers et événements organisés au Luxembourg, nous avons décidé de proposer une version adaptée à l'utilisation en classe. Après différents échanges et ateliers de conception avec des enseignants, la première version du Kniwwelino Classroom Kit (KCK) comprend plusieurs boites ("pupil box") pour travailler en binôme contenant : une carte électronique, des câbles de branchement et de nombreuses extensions électroniques (3 Diodes ÉlectroLuminescentes (DEL), un servo moteur, un bouton, un buzzer, des câbles), voir Figure 2. La versatilité du dispositif permet aux enfants d'imaginer et créer leurs propres projets.

Le KCK contient également du matériel pédagogique composé d'un manuel d'utilisation, de fiches d'activités supportant un apprentissage progressif des bases de la programmation et de l'électronique, des idées de projets technologiques transdisciplinaires expliqués pas-à-pas (par exemple une station météorologique), et des outils de créativité pour pousser les élèves à résoudre des problèmes et être créatifs comme les cartes mission, les cartes extension, une fiche de description de projet et les dés, voir Figure 3. Les cartes mission décrivent les différentes missions qui peuvent être confiées aux élèves comme point de départ pour développer leur propre projet Kniwwelino. Les cartes extension expliquent comment utiliser une extension en termes de branchement et de blocs à utiliser dans l'interface de programmation visuelle. Enfin les dés comprennent un dé indiquant les différentes missions et deux dés indiquant les différentes extensions. Les élèves lancent les dés et doivent trouver une idée pour résoudre la mission avec les deux extensions affichées par les dés.

\section{4 ÉTUDE D'ÉVALUATION SUR LE TERRAIN DU PREMIER PROTOTYPE DU KCK}

Dans cette section, nous présentons les questions de recherche qui ont guidé cette étude, les participants, le matériel utilisé, les indicateurs choisis ainsi que la procédure de test. Les résultats obtenus sont discutés dans la section suivante.

\subsection{Questions de recherche}

La présente étude a pour objet l'utilisation sur le terrain du KCK et pose les questions suivantes:

Q1 : Comment les enseignants s'approprient le KCK pour enseigner la PI en primaire et secondaire?

- Quels étaient les objectifs pédagogiques développés par les enseignants?

- Comment les enseignants ont-ils organisé les sessions d'apprentissage?

- Quelles pratiques pédagogiques ont-ils développées avec le KCK?

- Comment envisagent-ils de réutiliser le KCK dans le futur?

Q2 : Le KCK est-il adapté à l'enseignement de la PI en primaire et secondaire?

- Quelle est l'adéquation du matériel technologique et pédagogique proposés au besoin?

- Quelles compétences les élèves ont-ils pu développer avec le KCK?

- Quelle est la motivation des élèves à travailler avec le KCK ?

- Est-ce que le KCK suscite l'intérêt des élèves pour l'informatique?

- À quel point le KCK permet-il aux élèves d'être créatifs ?

Q3 : Quelle est la qualité perçue par les enseignants et les élèves de l'expérience utilisateur du KCK?

\subsection{Participants à l'étude}

4.2.1 Sélection des classes pilotes. La présente étude a été menée auprès de six écoles, sélectionnées pour représenter la diversité du tissu éducatif à Luxembourg : enseignement primaire et secondaire, écoles publiques et privées, enseignement en différentes langues (spécificité du Luxembourg), écoles distribuées sur le territoire national, voir le récapitulatif dans la Table 1.

4.2.2 Profils des enseignants ayant participé à l'étude. Onze enseignants ont participé à l'étude dont 7 hommes et 4 femmes sur 


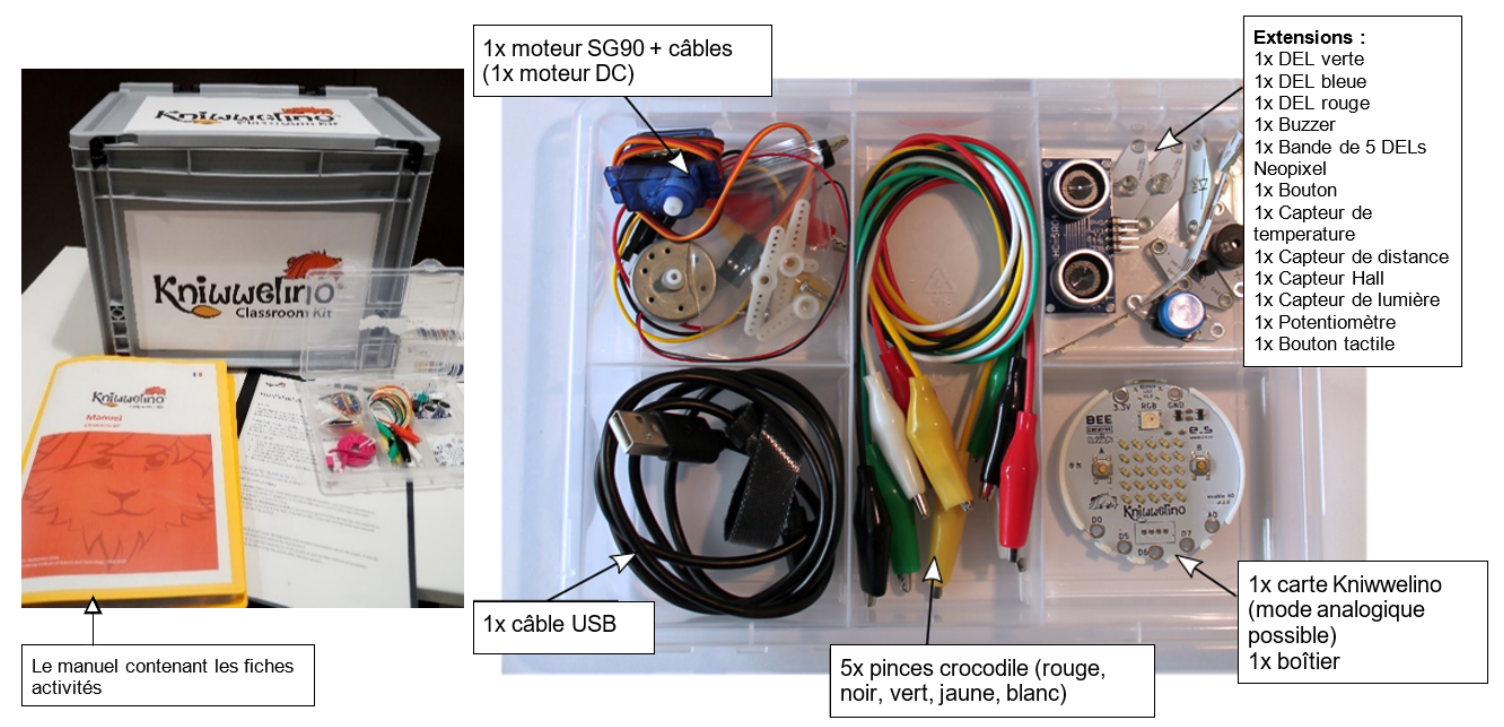

Figure 2: Le Kniwwelino Classroom Kit et son contenu.
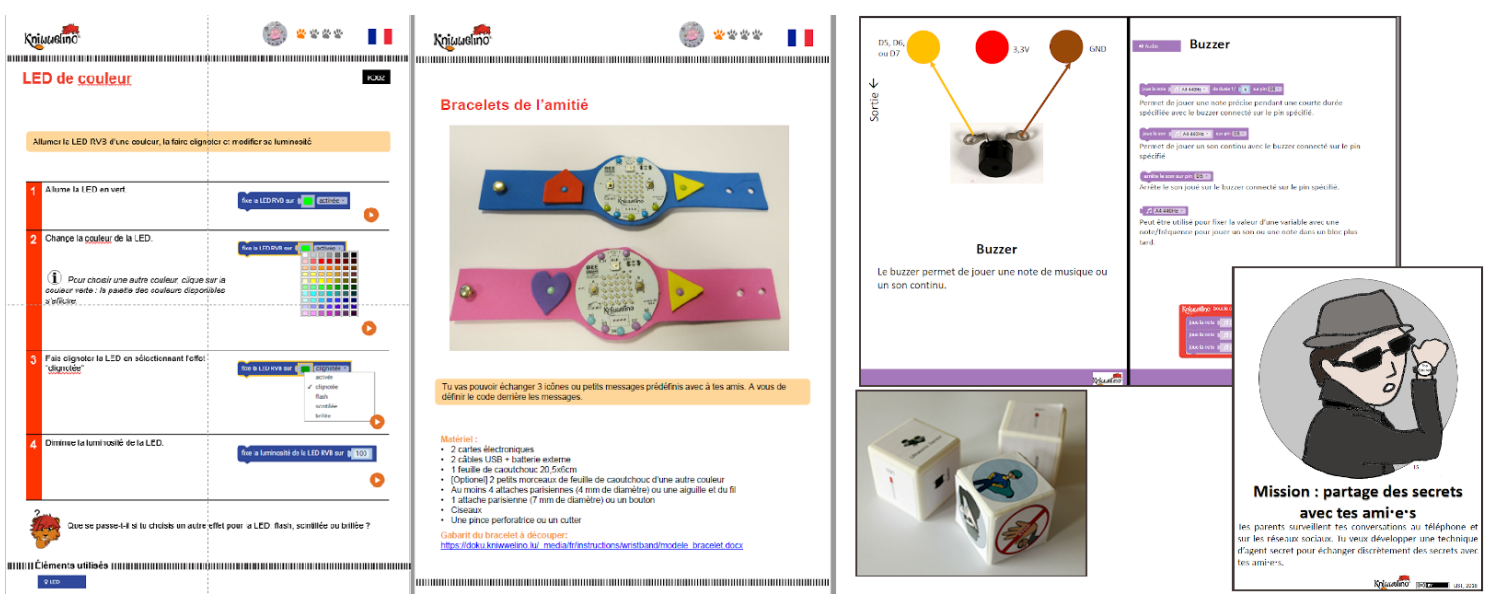

Figure 3: Matériel pédagogique : une fiche activité, une fiche projet, une fiche extension, les dés, une carte mission.

Table 1: Écoles pilotes de l'utilisation du KCK.

\begin{tabular}{|c|c|c|c|c|c|c|}
\hline École & Enseignement & Type & Localisation & Langue & Nombre d'élèves & Âge \\
\hline P1 & primaire & publique & Sud & français & 25 (1 classe & $9-11$ \\
\hline $\mathrm{P} 2$ & primaire & publique & capitale & luxembourgeois & $8(1$ classe $)$ & $10-12$ \\
\hline P3 & primaire & privé & capitale & anglais & $\begin{array}{l}90 \text { ( } 5 \text { classes de } 18 \\
\text { élèves }\end{array}$ & $9-11$ \\
\hline S1 & secondaire & publique & Sud & luxembourgeois & $\begin{array}{l}26 \text { (2 groupes de } \\
13 \text { élèves) }\end{array}$ & $14-15$ \\
\hline S2 & secondaire & publique & capitale & anglais, luxembourgeois & $\begin{array}{l}44 \text { ( } 2 \text { classes de } 22 \\
\text { élèves) }\end{array}$ & $12-13$ \\
\hline S3 & secondaire & privé & capitale & français & l & l \\
\hline
\end{tabular}

base du volontariat. Les enseignants participants ont majoritairement une expérience en enseignement de la PI, sauf à P1 et un des enseignants de P3. Cependant, à P3 une facilitatrice en enseignement des technologies accompagne les enseignants. Pour la suite 
des données, nous nous basons sur les 9 enseignants ayant complété leur profil. A propos de l'ancienneté de ces neuf enseignants : 4 enseignants ont plus de 20 ans d'ancienneté, 2 ont entre 10 et 20 ans d'ancienneté et 2 ont moins de 5 ans d'ancienneté. Avant l'utilisation de Kniwwelino, concernant leurs compétences en informatique : 4 enseignants estimaient avoir un niveau intermédiaire, 3 estimaient être compétents et 2 estimaient être experts (échelle utilisée : novice, intermédiaire, compétent, expert). Concernant la programmation avant l'utilisation de Kniwwelino : 3 enseignants se déclaraient novices, 3 avec un niveau intermédiaire, 1 estimait être compétent et 2 se déclaraient experts. Les outils cités ayant déjà été utilisés pour enseigner la PI et la programmation sont (entre parenthèses figure le nombre d'occurrences) : Scratch (6), Kniwwelino (5), Lego (4), code.org (4), Java (3), Arduino (2), Logo (2), Python (2), MySQL (1), Raspberry Pi (1) et Micro:bit (1).

4.2.3 Profils des élèves ayant participé à l'étude. 138 élèves (57 filles et 81 garçons, moyenne d'âge : 10,19 de 8 à 16 ans) des six écoles pilotes ont répondu à notre questionnaire pré-utilisation. La plupart des répondants déclarent avoir déjà programmé avant (76 de 137 répondants, soit 55,47\%), principalement à l'école (58 répondants) ou à la maison (33 répondants). Les élèves pensent que la programmation est amusante avec une moyenne de 4,47 (min 1; max 5). Et la majorité d'entre eux voudrait devenir développeur.se (3,85: $\min 1 \max 5)$. Une petite différence existe entre les garçons et les filles, mais rien de significatif ("c'est fun de programmer" : moyenne de 4,47 pour les garçons et 4,37 pour les filles; "devenir développeur.se" moyenne de 3,61 pour les garçons et 3,53 pour les filles). Il est important de noter que les élèves n'ont pas été sélectionnés.

\subsection{Matériel fourni}

Chaque école pilote a reçu un KCK, décrit en détail dans la Section 3, contenant 15 à 20 boîtes élève (voir Figure 2), un livret contenant du matériel pédagogique pour apprendre à développer à travers différentes activités et à réaliser des projets ainsi que du matériel pour supporter la créativité (voir Figure 3). Il était également possible d'emprunter un routeur $4 \mathrm{G}$, option que les écoles pilotes n'ont pas retenue au début de l'étude.

\subsection{Indicateurs et méthodes de collectes de données}

Pour collecter les informations nécessaires à cette étude, nous avons utilisé trois questionnaires, un journal de bord et un entretien semidirigé. Ils sont détaillés dans les sections suivantes.

fournal de bord. Les enseignants ont reçu un journal de bord à remplir à la fin de chaque session en la décrivant (types d'activités réalisées, apprentissage visé, matériel pédagogique et extensions utilisés, etc.) et en indiquant leurs observations.

Entretien avec les enseignants. Après la période d'utilisation en classe du KCK, nous avons réalisé un entretien semi-dirigé avec chaque enseignant. Cet entretien a permis de collecter des données qualitatives concernant : leurs objectifs et l'approche pédagogique mise en place, le déroulé réel des sessions et de l'apprentissage, les impacts sur les élèves de l'utilisation du KCK, leur opinion concernant le $\mathrm{KCK}$, leurs perspectives en termes de réutilisation et de transfert des compétences acquises.

Questionnaire UEQ pour les enseignants. Afin de mesurer l'expérience utilisateur fournie par le KCK, nous avons demandé aux enseignants de remplir le User Experience Questionnaire (UEQ)[14] après leur utilisation du KCK en classe. Ce questionnaire permet de mesurer l'attraction, la compréhensibilité, l'efficacité, la contrôlabilité, la stimulation et l'originalité. Nous étions particulièrement intéressés par la possibilité de mesurer les dimensions d'attraction et de qualité hédonique. Pour chaque échelle, plusieurs couples de mots opposés sont présentés. Le répondant doit indiquer lequel de ces deux mots correspond plus à son ressenti en utilisant une échelle de -3 à +3 , incluant le 0 qui est neutre.

Questionnaires pré-utilisation et post-utilisation pour les élèves. Nous avons demandé aux élèves de remplir deux questionnaires : le premier (pré-questionnaire) à remplir avant la présentation du $\mathrm{KCK}$, le second (post-questionnaire) à remplir après la formation dispensée par leurs enseignants. Les élèves pouvaient répondre aux deux questionnaires selon une échelle de Likert avec cinq items utilisant des émoticônes sourire comme recommandé pour les enfants de 9 à 11 ans par [9]. Les questionnaires avaient pour objectif de mesurer l'attitude des élèves vis-à-vis de la programmation en général et leur expérience utilisateur du KCK en particulier. Le pré-questionnaire permettait également d'avoir une vue sur la répartition démographique des participants à l'étude.

\subsection{Procédure de test}

Avant de mener l'expérimentation, nous avons invité les enseignants intéressés des écoles pilotes pour leur présenter Kniwwelino lors de deux ateliers de formation de quatre heures chacun. Le premier atelier était une prise en main de Kniwwelino avec la réalisation d'exercices, en suivant le matériel pédagogique fourni, pour apprendre à l'utiliser. Les enseignants ont reçu à cette occasion chacun une boîte élève pour essayer Kniwwelino dans leur école ou chez eux. Le second atelier était axé sur l'idéation de projets par les enseignants à réaliser avec leurs élèves avec des échanges entre participants et formateurs pour rendre le projet réalisable, ainsi que sur les retours des enseignants concernant les améliorations à apporter pour la boîte élève. Ces ateliers ont eu lieu cinq à sept mois avant l'utilisation sur le terrain, afin de laisser le temps aux enseignants de s'approprier l'outil et de maturer leur projet pédagogique. Sur les neuf enseignants ayant répondu au questionnaire, deux enseignants ont suivi uniquement le premier atelier, deux enseignants ont suivi uniquement le deuxième atelier, trois enseignants ont suivi les deux ateliers et les deux enseignants experts en informatique et programmation n'ont pas suivi les ateliers. Lors de ces ateliers, nous avons expliqué le rôle d'école pilote qui consiste à : (1) s'engager à délivrer au moins trois sessions de programmation avec ses élèves, libre aux enseignants de choisir le format et le contenu des sessions; (2) retourner les documents d'évaluation nécessaires à l'étude. La procédure de test était constituée de trois étapes synthétisées dans la Figure 4 :

(1) Au début de la première session : demander aux élèves de remplir le pré-questionnaire.

(2) A la fin de chaque session : remplir le journal de bord. 
(3) A la fin de l'utilisation du KCK en classe : demander aux élèves de remplir le post-questionnaire et de remplir pour leur part le questionnaire UEQ. De plus, les enseignants étaient invités à partager leur expérience via un entretien semi-dirigé.

4.5.1 Remarques concernant le SARS-CoV-2. L'étude a dû être interrompue à cause du confinement du au SARS-CoV-2. A la rédaction de cet article, sur les six écoles participant à l'étude, trois avaient fini leurs sessions avant le confinement, deux avaient commencé les sessions mais n'ont pas pu finir, et une n'a pas pu commencer. Les entretiens ont été menés en ligne avec les enseignants et l'analyse des questionnaires se base sur ceux disponibles en décembre 2020.

\section{RÉSULTATS DE L'ÉTUDE}

La présente section présente les résultats des questionnaires et l'analyse thématique des entretiens. Les résultats sont discutés selon les questions de recherches précisées précédemment, puis selon les résultats d'études de la littérature concernant des outils support à l'enseignement de la PI.

\subsection{Q1: Comment les enseignants s'approprient le KCK pour enseigner la PI en primaire et secondaire?}

5.1.1 Objectifs pédagogiques. Tous les enseignants ont utilisé le KCK en premier lieu pour initier leurs élèves à la PI et à la programmation. Mais ils ont également utilisé le KCK pour répondre à plusieurs sous-objectifs ou objectifs complémentaires, voir Table 3 :

- capacité à investiguer un sujet, à résoudre des problèmes et acquérir des compétences logiques. "My learning objective was to see if we could get 8 year-old to engage with codable device in a classroom setting. I wanted to see if we could get computational thinking which is not actually coding but understanding what a algorithm or variable is." (P3)

- initiation à la programmation pour préparer les élèves à l'utilisation de langages de programmation comme Python. "On utilise Kniwwelino blockly pour les cours inférieurs, en préparation de notre cours de langage de programmation en Python, on fera le lien vers Kniwwelino ou Scratch qu'ils ont eu en $5^{\circ} . "(\boldsymbol{S} 2)$ Voir de découvrir le débogage.

- initiation à l'électronique.

- développement de la capacité à collaborer et à s'entraider. "One of the big parts of this was that the kids were working together, and having to communicate and help each other. (...) They had to share their knowledge with their partner, and talk them through it. That's an added component to this, that's fantastic." (P3).

- développement des compétences de communication, notamment dans l'école $\mathbf{P} 2$ où il a été demandé aux élèves de documenter leur travail à travers des présentations faites aux parents. A S3 l'objectif d'acquérir des compétences de communication était fort avec la volonté de demander à des élèves de secondaire de transmettre l'utilisation du KCK à des élèves de primaire.

- gagner en autonomie.
- la créativité via la réalisation de projets ou l'expérimentation des options disponibles.

- susciter l'intérêt des enfants pour l'informatique.

5.1.2 Organisation des sessions d'apprentissage. Les écoles ont organisé différemment leurs sessions d'apprentissage, voir Table 2. A P1 et à P3 les élèves ont travaillé en binôme. A S1 les élèves étaient séparés en deux groupes : le premier a eu cours au premier semestre en enseignement mixte (à l'école et à distance à la maison), le deuxième a eu cours au second semestre et, à cause du confinement, n'a reçu que l'enseignement à distance à la maison. Les élèves ont d'abord travaillé seuls, puis en groupes pour créer leur projet. A S2 les classes étaient divisées en deux sous-groupes avec une session hebdomadaire d'une heure chacun et les élèves ont travaillé individuellement comme dans l'école P2.

Table 2: Organisation des sessions et matériel utilisé par les écoles pilotes. NA : Non applicable.

\begin{tabular}{lllll}
\hline Pilote & Seul ou binôme & $\begin{array}{l}\text { Nombre } \\
\text { de sessions }\end{array}$ & $\begin{array}{l}\text { Supports pédagogiques } \\
\text { utilisés }\end{array}$ & Matériel utilisé \\
\hline P1 & Binôme & 10 & $\begin{array}{l}\text { Feuilles d'activité } \\
\text { Feuilles d'activité }\end{array}$ & $\begin{array}{l}\text { Carte Kniwwelino } \\
\text { Carte Kniwwelino } \\
\text { + matériel de créativité }\end{array}$ \\
P2 & Seul & 9 & $\begin{array}{l}\text { Feuilles d'activité } \\
\text { Carte Kniwwelino }\end{array}$ \\
P3 & Binôme & $3-8$ & A développé son propre & Carte Kniwwelino \\
S1 & Seul puis en groupe & 11 & support pédagogique & + extensions \\
S2 & Seul & 7 & Feuilles d'activité & Carte Kniwwelino \\
S3 & NA & 0 & NA & NA \\
\hline
\end{tabular}

5.1.3 Pratiques pédagogiques observées. Selon les enseignants, la plupart des élèves n'avaient pas d'expérience en programmation, hormis quelques-uns ayant déjà programmé à de rares occasions (découverte via code.org à P3, découverte de Kniwwelino dans un événement à Luxembourg et quelques leçons de Scratch avec les élèves de P2). Ce qui est en opposition avec les déclarations des élèves qui estiment à $55 \%$ avoir déjà programmé et majoritairement à l'école. Les enseignants entendent probablement savoir programmer seul tandis que les élèves pensent peut-être avoir déjà été en contact avec un outil de programmation. Quatre écoles ont choisi une approche d'apprentissage progressive (P1, P2, S1 et S2). C'està-dire que dans un premier temps, ils ont enseigné les bases de la programmation (matrice, DELs, messages, variables et boucles). Ensuite ils ont expérimenté l'ajout d'extensions électroniques quand cela a été possible (DELs externes, buzzer, moteurs servo). Et finalement ils ont fait appel à la créativité des enfants en leur lançant un défi où ils ont fait appel à leur capacité de résolution de problèmes. Afin d'entraîner la PI, P3 a décidé de laisser les enfants libres de leur découverte de Kniwwelino et de la programmation en les guidant uniquement dans leur manière d'investiguer un sujet et de résoudre des problèmes.

"The idea was that the pupils apply computational thinking skills, such as you do one step at a time, programming is an iterative process, and if you try to map out the whole program at once, it's gonna be really hard to find your mistake. So if you put a block in, test it if it really works, and then you can add next block, 


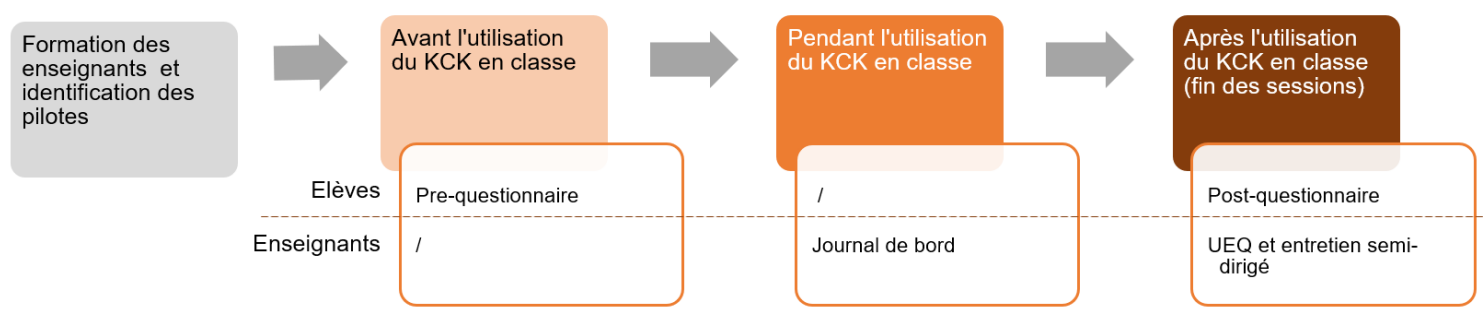

Figure 4: Protocole de l'étude.

and if it doesn't work it's really easy to fix your mistakes." (P3)

A S3 il était prévu d'utiliser le KCK avec des élèves de secondaire dans le cadre du makerlab (hors temps de cours, espace dédié à l'électronique, l'informatique et le bricolage) pour concevoir une maquette de maison connectée avec utilisation des extensions. Il était prévu que les élèves de secondaire une fois formés puissent enseigner la programmation à des élèves plus jeunes de primaire. Cela n'a pas pu se faire à cause du confinement.

Pour gagner en autonomie, $\mathbf{S} 2$ a développé un système de connexion de la carte Kniwwelino basée sur QR-code.

5.1.4 Réutilisation et transférabilité. Une partie importante de l'appropriation d'un outil est d'identifier si ses utilisateurs souhaitent continuer à l'utiliser et s'ils pensent que l'outil peut être transféré à d'autres. Tous les enseignants impliqués dans l'étude prévoient de réutiliser l'année prochaine le KCK (s'ils ont les mêmes classes). Les enseignants pensent que le KCK peut être utilisé en dehors de l'enseignement de la programmation dans des disciplines telles que les mathématiques, les sciences ou le français par exemple. De plus, d'autres enseignants des écoles pilotes ont montré leur intérêt pour l'utilisation du KCK lors de la prochaine année scolaire.

\begin{abstract}
"On a été jusqu'aux variables mais on a dû arrêter à cause du confinement. On va le réutiliser en $8^{\circ}$ et prendre le volet électronique avec (...) pour des projets beaucoup plus individuels avec les sensors." (S2)

"Il y a des petites passerelles : ça apprend la logique, ça peut avoir un lien avec les maths, les connecteurs logiques, ça peut avoir un lien avec les langues, le Français." (P1)
\end{abstract}

A propos de la transférabilité du KCK, les enseignants sont très satisfaits de la formation initiale à Kniwwelino et pensent qu'elle serait utile aux futurs enseignants utilisant le KCK. Cependant, ils soulignent également la complexité de la mise en place initiale de Kniwwelino. Les enseignants pensent que le succès dépend de l'expérience, de l'attitude et de l'état d'esprit de l'enseignant en matière de résolution de problèmes technologiques. Ils recommandent qu'un groupe d'enseignants agisse comme ressource pour les autres enseignants dans chaque école. L'expérience d'utilisation du KCK a tellement plu à P1 qu'ils ont lancé un projet avec quatre écoles en Europe pour explorer et promouvoir l'utilisation de Kniwwelino notamment à travers l'utilisation des Technologies de l'Information et de la Communication dans l'enseignement des langues, des mathématiques, des sciences et des arts pour des élèves du niveau primaire (9-10 ans).

\subsection{Q2 : Est-ce que le KCK est adapté à l'enseignement de la PI en primaire et secondaire?}

Selon les retours des enseignants dans les interviews, les enseignants ont vraiment apprécié l'utilisation du KCK dans leur classe. Et ils soulignent que les enfants ont adoré également l'expérience et se sont bien amusés. Ils ont fortement apprécié également le côté tangible de Kniwwelino. "C'est mieux que Scratch car on voit directement ce qu'on fait." (S1)

5.2.1 Retours concernant le matériel. Le matériel est adéquat à son utilisation en classe "Le kit est bien tel qu'il est pensé" (P1). Les boîtes sont faciles à transporter et à ranger. Les enseignants pensent que pour les plus jeunes élèves (fin maternelle, début primaire) les cartes suffisent. Ils pensent que les extensions demandent d'apprendre trop de nouvelles choses en même temps pour ce public. Les enseignants ont souligné qu'il est difficile de partager un KCK entre plusieurs classes de la même école parce que cela demande beaucoup de coordination entre les enseignants. Certains enseignants ont été confrontés à des cartes défectueuses (P1, P2, P3). En fait, les cartes ne pouvaient pas redémarrer proprement parce que les élèves les débranchaient et les rebranchaient trop vite. Le problème a été réglé en réinitialisant les cartes grâce à l'outil développé dans ce but. Deux enseignants ont souligné des problèmes liés aux câbles : des câbles qui cassent trop vite quand ils sont manipulés par les enfants (P2) ou des élèves qui trouvaient trop dur l'utilisation des pinces crocodiles puisqu'ils ne connaissaient que les câbles USB (S1).

5.2.2 Retours concernant l'interface de programmation visuelle. L'interface de programmation visuelle a été jugée facile à utiliser, propre, simple et colorée. "It's clean and really simple, I love the little tutorials that pop up." (P3) Un enseignant de $\mathbf{S} 1$ a souligné que l'interface de programmation visuelle permet aux enfants de capter la logique de programmation. Les élèves ont l'impression de jouer avec un puzzle. "Une introduction à la programmation avec un feedback en hardware, c'est une approche qui est très bien pour les élèves." (S2) Les enseignants notent cependant que le manque de retour direct dans l'interface de programmation visuelle est inhabituel pour les élèves. "in code.org you have instant feedback (cheering and clapping). In real programming you don't have that. The only feedback is whether or not it works. When it doesn't it can be frustrating. 
We had to make this clear in the sessions, and then we experienced real success." (P3)

5.2.3 Retours concernant l'utilisation du Wi-Fi. Beaucoup de problèmes de connexion au Wi-Fi ont été rencontrés. Ces difficultés étaient principalement dues aux infrastructures techniques des écoles qui sont très protégées ou au fait que le Wi-Fi n'est pas assez puissant dans certaines salles de classes. Cela a entraîné des problèmes de téléchargement et de la frustration pour les enfants. Pour résoudre ces problèmes, trois écoles ont développé leur propre réseau dédié à Kniwwelino et deux écoles ont finalement emprunté le routeur $4 \mathrm{G}$ proposé au départ. Les enseignants ont également reporté que le téléchargement du code sur les cartes via le $\mathrm{Wi}-\mathrm{Fi}$ peut être lent et générer des frustrations.

5.2.4 Retours concernant les supports pédagogiques. Le manuel fourni dans le KCK a été jugé bien adapté, utile et très clair. Il a permis aux enseignants de démarrer facilement leurs activités. Les enseignants ont principalement utilisé la version papier. Quelquesuns ont adapté les fiches d'activités avant de les imprimer. Notamment, certains enseignants ont complété l'approche proposée dans le KCK en ajoutant des exercices de débogage et de vérification de code.

"Le petit classeur aide bien à démarrer." (P1)

"Les supports que vous avez fournis étaient très aidant. On a utilisé le handbook et les tutoriels sur le wiki. On les adaptés au fur et à mesure."

(S2)

"Exercise sheets are great and easy to copy."

(P3)

À P3 les enseignants ont utilisé code.org au début, puis ils ont mis en application le savoir acquis avec le KCK. Les fiches d'activités étaient disponibles pour consultation par les élèves. A S1, l'enseignant a développé son propre curriculum composé de 75 leçons progressives : 50 de programmation et 25 d'électronique.

5.2.5 Compétences développées par les élèves avec le KCK. L'utilisation du KCK par les écoles pilotes a permis d'identifier en moyenne le développement de compétences des élèves au niveau de la programmation et l'électronique, la résolution de problèmes et la logique, la créativité, la collaboration et l'autonomisation, voir Table 3. P3-E4 n'a pas pu avancer beaucoup à cause du confinement qui a mis fin aux séances et S3-E9 n'a pas pu commencer. Les sections suivantes développent les observations des enseignants sur les compétences développées sur base des interviews. En plus des compétences listées dans le tableau, P3 a également noté une amélioration de la persévérance des élèves. "Perseverance. Sticking with a problem and trying to solve it." (P3)

Apprendre la programmation et électronique. Tous les enseignants ont observé que les élèves ont amélioré leurs compétences en programmation et électronique, même s'ils n'ont pas toujours atteint leur objectif final. En particulier, ils ont observé qu'ils ont intégré la logique de programmation. "Ils partaient de rien, et après il savaient manipuler, trouver les erreurs avec le feedback (...) Ils comprenaient la différence entre "au démarrage" et "en répétition continue", c'est le plus important au départ." (S2) Les enseignants ont également observé que les élèves ont commencé à apprendre puis adopter le vocabulaire de la programmation, comme dans [8] : "Once they had those words available to them and could see them, they started to use them a lot more." (P3)

Apprendre la résolution de problèmes. Le pilote P3, qui a orienté particulièrement son enseignement sur la résolution de problèmes, a observé une progression importante concernant cette compétence ainsi que le développement de leur persévérance et leurs compétences d'investigation. "It was a huge enquiry for the students. An enquiry in how they could be problem solvers and what they could get the Kniwwelino to do." (P3) Mais les autres écoles pilotes ont également observé que les élèves devenaient de plus en plus autonomes dans la recherche de solutions à leurs problèmes. "Ils ont eu des soucis, donc ils ont commencé un petit peu à chercher des solutions. Même si c'étaient des soucis techniques au départ, parfois ils cherchaient aussi leurs propres solutions pour ça, ça c'était pas inintéressant." (S1)

Apprendre la créativité. Les enseignants ont pu observer la créativité s'exprimer principalement en ce qui concerne l'exploration des différents éléments de Kniwwelino. Ils ont vu les élèves essayer différentes couleurs et afficher différents symboles sur la carte. A $\mathbf{P 1}$, la créativité n'a pas pu être explorée à cause du confinement. A (P2), l'enseignant a pointé que ses élèves ont imaginé des projets trop complexes qu'ils n'ont pas réussi à développer, ce qui les a frustrés.

"On voyait qu'ils développaient leur esprit créatif. Il faut leur laisser l'espace pour. Dès qu'on ouvre un peu, la créativité commence." (S2)

"Some of my kids got really into it. It showed them that they could be creative with a device like that." (P3)

Apprendre la collaboration et les interactions sociales. Les écoles pilotes ont observé qu'avec l'utilisation du KCK, et le fait de travailler en binôme, les compétences en termes de collaboration et d'interaction sociales se sont améliorées. Ceci est dû également à la possibilité de s'envoyer des messages et l'aide que les élèves se sont spontanément apportée. "Le partage a commencé surtout avec l'envoi de messages." (S2)

5.2.6 Motivation des élèves. Deux écoles (S2 et P1) mentionnent que les élèves étaient peu motivés au début mais se sont intéressés au fur et à mesure de l'avancement. P1 justifie cela par le fait que la progression était très lente au début, mais une fois les problèmes techniques maîtrisés, les élèves avaient plus de temps pour programmer. De plus, dès que les élèves étaient capables de maîtriser plusieurs éléments de Kniwwelino, ils se sont amusés et ont joué avec. Idem chez $\mathbf{P} 2$, où il $\mathrm{y}$ a eu beaucoup de problèmes techniques au début qui ont impacté négativement l'expérience des élèves qui étaient pourtant très motivés, mais après la résolution des problèmes techniques les élèves se sont amusés.

"Il y a des élèves qui ont commencé avec beaucoup de peur, mais dès qu'ils ont fait le premier pas, ils y ont pris goût, surtout les filles." (S2) "Au début c'est vrai qu'il y avait pas une grosse grosse motivation, sûrement en rapport un peu avec ça (les problèmes de connexion au réseau de l'école) aussi. Puis au fur à mesure qu'ils 
Table 3: Objectifs des enseignants et compétences acquises par les élèves selon les enseignants. Légende : $\square$ objectif acquis, $\square$ objectif non acquis, $\star$ n'était pas un objectif mais a été acquis, / n'était pas un objectif.

\begin{tabular}{|c|c|c|c|c|c|c|c|c|c|}
\hline Objectif / Enseignant & P1-E1 & $\mathrm{P} 2-\mathrm{E} 2$ & P3-E3 & P3-E4 & P3-E5 & S1-E6 & S2-E7 & S2-E8 & S3-E9 \\
\hline PI & $\mathbf{\square}$ & घ & $\mathbf{\square}$ & $\square$ & - & $\mathbf{\square}$ & घ & $\mathbf{\square}$ & / \\
\hline Programmation & - & घ & $\star$ & $\square$ & $\square$ & - & / & - & $\square$ \\
\hline Électronique & घ & / & / & $\square$ & / & $\square$ & / & $\square$ & / \\
\hline Résolution de problèmes & $\boldsymbol{\square}$ & घ & घ & $\square$ & - & $\boldsymbol{\square}$ & $\boldsymbol{\square}$ & घ & $\square$ \\
\hline Logique & $\boldsymbol{\square}$ & $\mathbf{\square}$ & $\square$ & $\square$ & - & $\square$ & $\mathbf{\square}$ & घ & $\square$ \\
\hline Créativité & $\square$ & घ & घ & $\square$ & घ & घ & घ & घ & $\square$ \\
\hline Collaboration & घ & $\square$ & घ & $\square$ & घ & घ & l & घ & $\square$ \\
\hline Communication & घ & $\square$ & - & $\square$ & घ & $\mathbf{\square}$ & / & घ & $\square$ \\
\hline Autonomisation & / & घ & घ & $\square$ & घ & l & / & घ & $\square$ \\
\hline $\begin{array}{l}\text { Susciter l'intérêt pour les } \\
\text { carrières informatique }\end{array}$ & Non & Oui & Oui & Non & Non & Oui & Oui & Oui & Oui \\
\hline Gestion du confinement & Arrêt & Arrêt & Fini avant & Arrêt & Fini avant & A continué & Arrêt & A continué & Arrêt \\
\hline
\end{tabular}

ont commencé à mieux maîtriser, pour pouvoir explorer un peu plus eux-mêmes, là ils étaient plus motivés, ça bougeait plus." (P1)

"Au départ aussi c'était assez limité, quand on fait les premières séances, où il y a qu'un type de programme à faire, vous travaillez sur un élément, bon voilà, ils ont vite fait le tour. Mais quand on commence à intégrer plus d'éléments dedans, du coup ils peuvent plus jouer et créer des choses et du coup cela les intéresse plus." (P1)

Pour P3 Au début les élèves ont connu beaucoup de frustration parce qu'ils manquaient de retour direct d'information dans l'interface de programmation visuelle : ils s'attendaient à savoir directement dans l'interface si leur programme était juste ou non, comme cela est fait dans code.org ou Scratch. Puis ils ont commencé à s'amuser surtout quand ils ont découvert l'échange de messages. D'après l'observation des enseignants, les élèves ont particulièrement apprécié l'approche ludique qui les a beaucoup motivés et le fait qu'ils puissent progressivement apprendre. "Les élèves aiment le côté ludique, l'approche par le jeu. Ils aiment la progression, "on avance, c'est cool". L'apprentissage est facile et logique." (S1) Les activités préférées des élèves étaient : changer la couleur des DELs, afficher des icônes sur la matrice de DELs comme des émoticônes, créer des animations et échanger des messages.

5.2.7 Gestion de la crise sanitaire. Sur les neuf enseignants ayant répondu, deux avaient fini leurs sessions avec Kniwwelino avant le confinement dû au SARS-CoV-2, cinq enseignants ont simplement arrêté l'enseignement avec Kniwwelino et deux ont continué, voir Table 3. Ceux qui ont continué (S1-E6 et S2-E8) sont des enseignants du secondaire. Ils ont pu fournir une carte Kniwwelino à chaque élève pour qu'ils puissent continuer à travailler à la maison. Les élèves ont reçu des instructions pour pouvoir connecter leur carte Kniwwelino à leur ordinateur ou tablette. Puis les élèves ont reçu du support de la part des enseignants via Teams quand cela était nécessaire.
5.2.8 Susciter l'intérêt des élèves pour l'informatique. D'après le post-questionnaire distribué aux élèves ayant participé à l'étude pilote, la grande majorité d'entre eux veulent : devenir développeu-rs·ses (59,14\%), développer d'autres projets avec Kniwwelino $(67,39 \%)$ et essayer d'autres outils de programmation $(85,87 \%)$, voir Figure 5. Ils trouvent la programmation plutôt amusante $(87,23 \%)$.

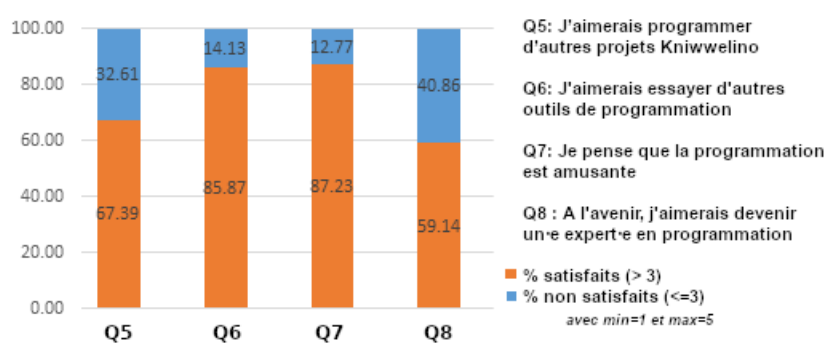

Figure 5: Intérêt des élèves pour la programmation à la suite de leur utilisation du KCK en classe.

\subsection{Q3: Comment les enseignants et les élèves perçoivent-ils l'expérience utilisateur du KCK ?}

5.3.1 L'expérience utilisateur des enseignants. L'analyse des résultats du questionnaire UEQ concernant la première version du prototype du KCK montre des valeurs positives pour toutes les échelles : attractivité (moyenne 1,596 avec min -3 max +3 et écart type 0,71), compréhensibilité $(1,194$ - 0,85), efficacité $(1,028$ - 0,59), contrôlabilité $(1,222$ - 0,76), simulation $(2,0-0,66)$ et originalité $(1,667-0,81)$. On peut en conclure que le prototype donne une première bonne impression en termes d'expérience utilisateur. Cependant, l'efficacité doit être améliorée. L'échelle stimulation a quant à elle un très bon score de 2. Dans la Figure 6, nous présentons les moyennes obtenues (minimum -3, maximum 3) pour chaque item du questionnaire UEQ. Les items intéressant $(2,4)$, apporte de la valeur $(2,3)$ 


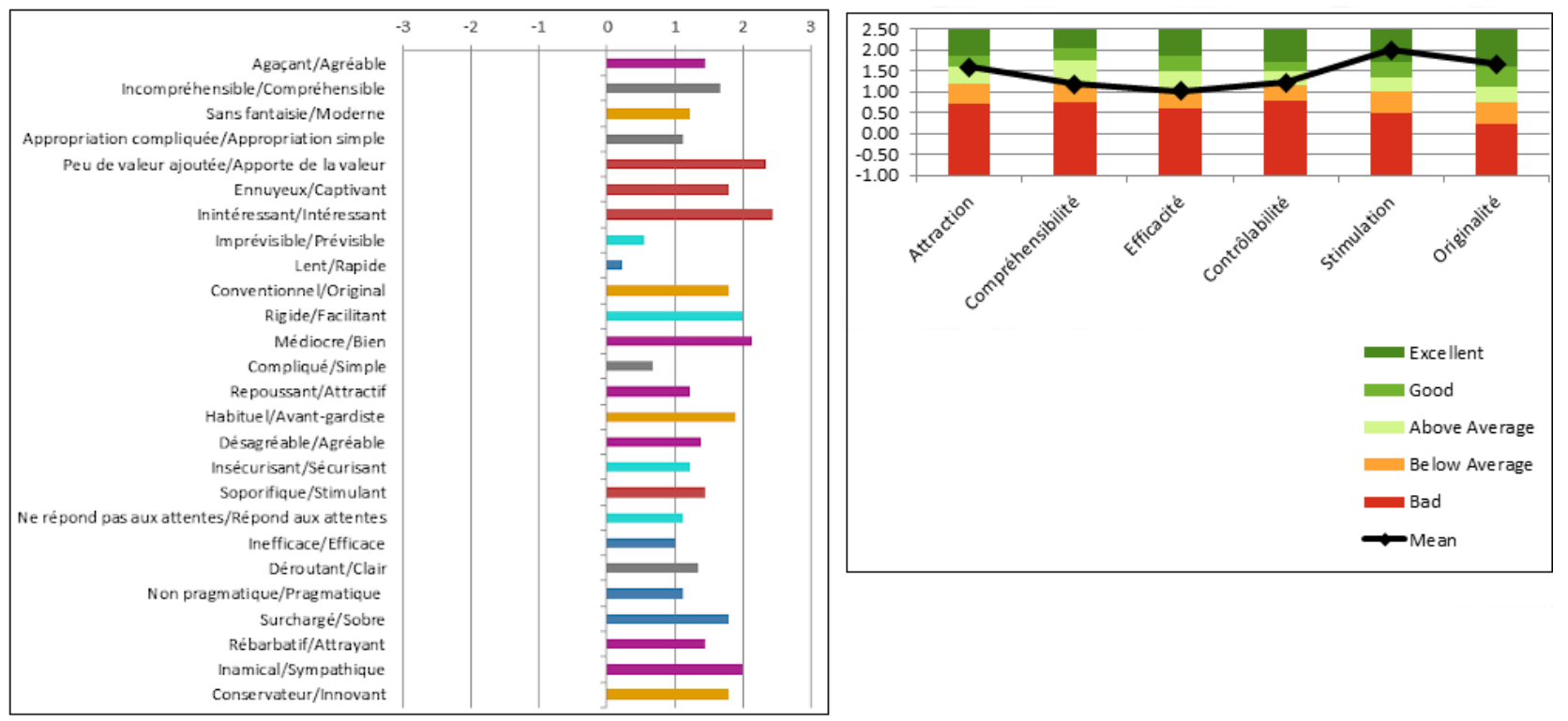

Figure 6: A gauche : résultats des réponses des enseignants au questionnaire UEQ concernant la première version du prototype du KCK, par items. - A droite : comparaison du KCK à la base de référence fournie par UEQ.

et bien $(2,1)$ ont obtenus les meilleurs résultats. Les items simple $(0,7)$, prévisible $(0,6)$ et rapide $(0,2)$ ont obtenus les moins bons scores. Mais nous pouvons noter que pour tous les couple d'items, le résultat reste toujours positif.

Le questionnaire UEQ permet également de comparer un nouveau produit en termes d'expérience utilisateur à un large échantillon d'autres produits largement utilisés. Cette base de données est divisée en cinq catégories : Excellent, Bien, Au-dessus de la moyenne, En dessous de la moyenne et Mauvais [21]. La Figure 6 montre la position du KCK par rapport aux éléments de cette base de référence. Le KCK est : excellent concernant la stimulation et l'originalité, au-dessus de la moyenne concernant l'attraction et la contrôlabilité, en dessous de la moyenne concernant la compréhensibilité et l'efficacité. Aucune échelle n'est dans la partie mauvais.

5.3.2 L'expérience utilisateur des élèves. 94 élèves ont répondu au questionnaire post-utilisation du KCK en classe. Les résultats montrent que les élèves sont satisfaits de leur utilisation : 82,98\% aiment programmer avec Kniwwelino, 86,02\% trouvent excitant de programmer avec Kniwwelino, $81,72 \%$ pensent qu'il est facile de programmer avec Kniwwelino et 76,6\% pensent que tout le monde peut programmer avec Kniwwelino, voir Figure 7.

\subsection{Discussion des résultats de l'étude par rapport à d'autres outils de support de l'enseignement de la pensée informatique}

Comme dans notre étude, l'étude de Scratch par Kaučič concerne des élèves de primaire et secondaire [13]. Pour répondre au questionnaire de Kaučič, les élèves ont évalué les items sur une échelle de Likert allant de 1 (tout à fait en désaccord) à 5 (tout à fait d'accord). Si nous essayons de comparer avec les résultats de Kaučič pour

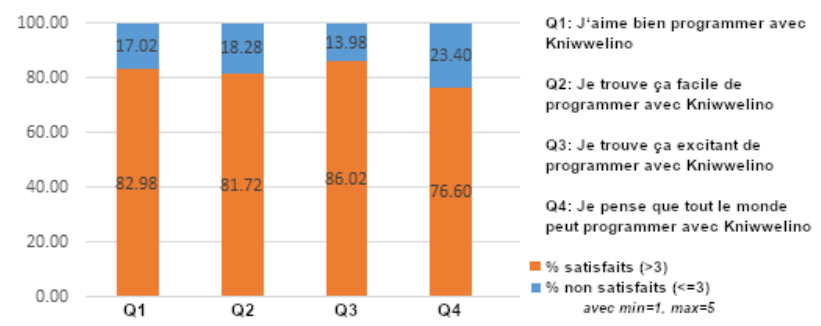

Figure 7: Satisfaction des élèves concernant leur expérience d'utilisation du KCK en classe.

Scratch (voir Table 4), nous pouvons observer que les élèves apprécient de programmer avec Kniwwelino (3,90 par rapport à 3,81 pour Scratch). Scratch semble plus facile à utiliser pour la programmation (3,94 contre 3,62 pour Kniwwelino) et un peu plus amusant $(4,03$ contre 3,90 pour Kniwwelino). Cependant, la comparaison doit être interprétée avec précaution : les questions ne sont pas exactement les mêmes, le nombre de participants n'est pas le même, et même si les contextes sont similaires, ils ne sont pas les mêmes. Néanmoins, elle montre que Kniwwelino a fourni une expérience similaire aux élèves.

Dans l'étude de Shina sur la découverte de la programmation avec Scratch, $60 \%$ des élèves interrogés ont trouvé facile d'utiliser Scratch [20]. En comparaison, pour Kniwwelino, dans cette étude, le pourcentage d'élèves satisfaits concernant la facilité d'utilisation de Kniwwelino est de 80,65\% (voir Figure 7).

Enfin, dans l'étude menée par Gibson, BBC Micro:bit a été évalué par des élèves de deux écoles primaires pendant un ou deux jours d'utilisation [7]. Les élèves ont évalué le plaisir d'utiliser BBC Micro:bit grâce à une échelle de Likert allant de 1 (très ennuyeux) 
à 5 (très agréable). $92 \%$ des élèves de l'école $\mathrm{A}$ et $90 \%$ des élèves de l'école $\mathrm{B}$ ont déclaré que l'utilisation de BBC Micro:bit était soit agréable, soit très agréable. Les résultats du BBC Micro:bit sont un peu meilleurs que ceux obtenus à la question "Je trouve ça excitant de programmer avec Kniwwelino" dans notre étude, où $86 \%$ étaient excités ou très excités (voir Figure 7). Mais, encore une fois, les résultats ne peuvent pas être comparés complètement.

Table 4: Comparaison entre l'étude de Kaučič avec Scratch [13] et notre étude avec le KCK.

\begin{tabular}{|c|c|c|c|}
\hline \multirow{2}{*}{$\begin{array}{l}\text { Etude de Kaučič avec Scratch } \\
\text { Item (et traduction) }\end{array}$} & \multirow[b]{2}{*}{ Score } & \multicolumn{2}{|l|}{ Notre étude avec le KCK } \\
\hline & & Item & Score \\
\hline $\begin{array}{l}\text { Scratch is attractive (I like it) } \\
\text { Scratch est intéressant (j'aime bien) }\end{array}$ & 3,81 & $\begin{array}{l}\text { J'aime bien programmer avec } \\
\text { Kniwwelino }\end{array}$ & 3,90 \\
\hline $\begin{array}{l}\text { Scratch is easy to use } \\
\text { Scratch est facile à utiliser }\end{array}$ & 3,94 & $\begin{array}{l}\text { Je trouve ça facile de programmer } \\
\text { avec Kniwwelino. }\end{array}$ & 3,62 \\
\hline $\begin{array}{l}\text { Scratch was fun to work with } \\
\text { C'était amusant de travailler avec Scratch }\end{array}$ & 4,03 & $\begin{array}{l}\text { Je trouve ça excitant de programmer } \\
\text { avec Kniwwelino }\end{array}$ & 3,90 \\
\hline
\end{tabular}

\section{CONCLUSION}

Le KCK a été utilisé avec succès dans différents contextes scolaires : en primaire et secondaire, pour initier à la programmation, à la PI et à la résolution de problèmes, avec et sans utilisation des extensions, en utilisant le curriculum proposé ou en développant son propre curriculum, en binômes ou seul. Ce qui montre la force du KCK : sa versatilité. L'expérience utilisateur est globalement positive pour les élèves et pour les enseignants et les résultats du UEQ sont plutôt encourageant pour un premier prototype.

Les impacts sur les élèves sont multiples. Les connaissances et compétences en programmation, en résolution de problèmes, en créativité et en collaboration ont progressé. Cependant, nous pouvons nous demander si la découverte d'une nouvelle activité comme la programmation n'a pas à elle seule suscité l'intérêt des élèves. Un autre impact, totalement inattendu, est la réduction de l'écart entre les genres en programmation. Certains enseignants ont pointé que le KCK est attrayant pour les filles et les garçons. "Dès que ça devient créatif, on a les filles avec." (S2)

Les enseignants pensent que le Kniwwelino Classroom Kit est parfait pour s'initier à la programmation et ils le recommanderaient à leurs collègues pour donner un premier contact à leurs élèves avec la pensée informatique et la logique, avant d'utiliser des langages de programmation.

Les améliorations à envisager sont : faciliter l'installation et la mise en place, le temps de chargement du code sur la carte et la robustesse. Les points forts du KCK sont : le caractère tangible de la plateforme d'apprentissage, la possibilité offerte, notamment grâce à la matrice et aux DELs, de pousser à la créativité, ainsi que le caractère social grâce au travail en binôme et l'échange de messages.

\section{REMERCIEMENTS}

Nous remercions les écoles pilotes et les enseignants qui ont eu le courage de tenter l'aventure et dont l'engagement et l'enthousiasme ont grandement contribué au succès de cette expérience. Cette étude a été financée par le FNR.

\section{RÉFÉRENCES}

[1] Charoula Angeli, Joke Voogt, Andrew Fluck, Mary Webb, Margaret Cox, Joyce Malyn-Smith, and Jason Zagami. 2016. A K-6 computational thinking curriculum framework: Implications for teacher knowledge. Fournal of Educational Technology \& Society 19, 3 (2016), 47-57.

[2] Arduino. accessed September 30, 2020. https://www.arduino.cc/

[3] BBC. accessed September 30, 2020. BBC Micro:bit. http://microbit.org/

[4] Ayah Bdeir. 2009. Electronics as material: littleBits. In Proceedings of the 3rd International Conference on Tangible and Embedded Interaction. 397-400.

[5] Calliope. accessed September 30, 2020. https://calliope.cc/

[6] Yu-Hui Ching, Yu-Chang Hsu, and Sally Baldwin. 2018. Developing computational thinking with educational technologies for young learners. TechTrends 62 , 6 (2018), 563-573.

[7] Seanpaul Gibson and Patrick Bradley. 2017. A study of Northern Ireland Key Stage 2 pupils' perceptions of using the BBC Micro: bit in STEM education. The STeP fournal 4, 1 (2017), 15-41.

[8] Shuchi Grover. 2011. Robotics and engineering for middle and high school students to develop computational thinking. In annual meeting of the American educational research association, New Orleans, LA.

[9] Lynne Hall, Colette Hume, and Sarah Tazzyman. 2016. Five degrees of happiness: Effective smiley face likert scales for evaluating with children. In Proceedings of the The 15th International Conference on Interaction Design and Children. 311-321.

[10] Ting-Chia Hsu, Shao-Chen Chang, and Yu-Ting Hung. 2018. How to learn and how to teach computational thinking: Suggestions based on a review of the literature. Computers \& Education 126 (2018), 296-310.

[11] Rose Johnson, Venus Shum, Yvonne Rogers, and Nicolai Marquardt. 2016. Make or shake: An empirical study of the value of making in learning about computing technology. In Proceedings of the The 15th International Conference on Interaction Design and Children. 440-451.

[12] Eva-Sophie Katterfeldt, Mutlu Cukurova, Daniel Spikol, and David Cuartielles. 2018. Physical computing with plug-and-play toolkits: Key recommendations for collaborative learning implementations. International fournal of Child-Computer Interaction 17 (2018), 72-82.

[13] B Kaučič and T Asič. 2011. Improving introductory programming with Scratch?. In 2011 Proceedings of the 34th International Convention MIPRO. IEEE, 1095-1100.

[14] Bettina Laugwitz, Theo Held, and Martin Schrepp. 2008. Construction and evaluation of a user experience questionnaire. In Symposium of the Austrian HCI and usability engineering group. Springer, 63-76.

[15] Lego. accessed September 30, 2020. Mindstorms. https://www.lego.com/enus/themes/mindstorms

[16] Sze Yee Lye and Joyce Hwee Ling Koh. 2014. Review on teaching and learning of computational thinking through programming: What is next for K-12? Computers in Human Behavior 41 (2014), 51-61.

[17] Valérie Maquil, Christian Moll, Lou Schwartz, and Johannes Hermen. 2018. Kniwwelino: A Lightweight and WiFi Enabled Prototyping Platform for Children. In Proceedings of the Twelfth International Conference on Tangible, Embedded, and Embodied Interaction. 94-100.

[18] José-Manuel Sáez-López, Marcos Román-González, and Esteban Vázquez-Cano. 2016. Visual programming languages integrated across the curriculum in elementary school: A two year case study using "Scratch" in five schools. Computers \& Education 97 (2016), 129-141.

[19] Ronny Scherer, Fazilat Siddiq, and Bárbara Sánchez Viveros. 2020. A metaanalysis of teaching and learning computer programming: Effective instructional approaches and conditions. Computers in Human Behavior (2020), 106349.

[20] Despoina Schina, Vanessa Esteve González, and Mireia Usart Rodríguez. 2019. Gender differences in students' feedback and performance in Scratch programming. In Conference Proceedings EDUNOVATIC 2018: 3rd Virtual International Conference on Education, Innovation and ICT. Adaya Press, 36.

[21] Martin Schrepp, Andreas Hinderks, and Jörg Thomaschewski. 2014. Applying the user experience questionnaire (UEQ) in different evaluation scenarios. In International Conference of Design, User Experience, and Usability. Springer, 383392.

[22] Sue Sentance, Jane Waite, Steve Hodges, Emily MacLeod, and Lucy Yeomans. 2017. " Creating Cool Stuff" Pupils' Experience of the BBC micro: bit. In Proceedings of the 2017 ACM SIGCSE technical symposium on computer science education. 531-536.

[23] Jeannette Wing. 2017. Computational thinking's influence on research and education for all. Italian fournal of Educational Technology 25, 2 (2017), 7-14. 